\title{
Patología dual: la nosología incierta. Narrativas profesionales sobre un cajón de sastre
}

\author{
Natàlia Lledó Carceller Maicas \\ Máster en Antropología de la Medicina y Salud Internacional, URV \\ natalia.carceller@urv.cat
}

Resumen: El sistema de salud mental catalán ba experimentado un proceso de evolución durante el cual se han producido cambios significativos que han conformado el modelo vigente. A pesar de los avances, existen aún ámbitos en los que las carencias se hacen patentes, como es el caso de la unificación de las redes de salud mental y drogadicciones. La Patología Dual se erige en el punto clave a partir del cual analizar esta situación, puesto que esta "nueva" categoría diagnóstica, que implica la presencia de un trastorno mental y un trastorno adictivo de manera concomitante, nos permite ver el modo en que ambas redes se relacionan. La paradoja reside en el hecho de que dicha categoría no está presente en los manuales diagnósticos, y a pesar de eso es empleada en la práctica diaria de los profesionales. A partir del análisis de las narrativas de los profesionales en el contexto sanitario catalán se observa la falta de consenso en torno a la definición de Patología Dual, la cual constituye un gran cajón de sastre.

Palabras clave: Patología Dual, salud mental, drogadicciones, narrativas.

Abstract: The Catalan mental health system has undergone a process of evolution during which significant changes have been made to constitute the model currently in force. In spite of the advances that have been made, there are still areas in which there are clear shortcomings: for example, the joining of the networks of mental health and drug-addictions into a single network. The Dual Pathology is fundamental to the analysis of this situation, since this "new" diagnostic category, which involves the concomitant presence of a mental disorder and an addictive disorder, makes I t possible to see how the two networks are related. The paradox Is, however, that this category is not present in the diagnostic manuals, but It is still very much part of professional daily practice. The analysis of the narratives of health professionals in the Catalan context reveal a lack of consensus about the definition of Dual Pathology, which has become a ragbag of numerous classifications.

Keywords: Dual Pathology, mental health, drug-addictions, narratives. 


\section{Introducción}

Dentro del sobresaturado sistema diagnóstico psiquiátrico actual, el que nos ha ido proveyendo durante largo tiempo de múltiples y variadas etiquetas con las cuales poder "identificarnos" o "identificar" a los otros, ha surgido "recientemente" una "nueva" nosología que ha permitido, de un modo informal, unificar dos campos que llevan años intentado unirse: la salud mental y la drogodependencia. Así, se ha configurado lo que hoy en día se conoce como la "Patología Dual".

Esta categoría, no existente dentro de los referentes del diagnóstico psiquiátrico como el DSM-IV-TR y el CIE-10, hace referencia a los pacientes que presentan un trastorno mental y un trastorno por abuso de sustancias al mismo tiempo, constituyéndose así como un gran cajón de sastre donde reina la ambigüedad y escasea el consenso.

La finalidad que persigo en el presente artículo es definir cuáles son los discursos y definiciones que encontramos actualmente en los profesionales que se dedican a la atención especializada al colectivo de pacientes duales en Cataluña, con el objetivo de poder analizar hasta qué punto existe o no consenso en torno a la categoría de "Patología Dual", y cuáles son los puntos de convergencia y divergencia a partir del análisis de las narrativas de los profesionales. Pretendo también, en última instancia, incitar a la reflexión y al debate sobre la atención en salud.

\section{Metodología}

Para realizar la presente investigación se ha llevado a cabo un trabajo de campo de nueve meses de duración, entre octubre de 2010 y junio de 2011. La metodología empleada en la investigación se basa en un enfoque holístico que nos permite analizar la temática de forma global, ya que posibilita el diálogo entre los niveles macro y microsocial. Este enfoque resulta de gran utilidad científica, puesto que permite la descripción y el análisis de las cosas de forma concreta, facilitándonos así el extraer hipótesis, reflexiones, etc.

Debido a que no existen demasiados datos en torno a esta temática de investigación —es un ámbito sobre el que se ha estudiado poco-, era necesario realizar un trabajo de campo exhaustivo que permitiera recopilar y crear datos con los que poder trabajar y realizar el pertinente análisis. 
El empleo de la etnografía como metodología de aproximación a la realidad se consideró necesario ya que, al implicar una relación continuada e intensa con el grupo estudiado, permitía acceder a los datos del nivel microsocial de manera inmediata. Así, se utilizó en combinación con determinadas técnicas cualitativas, como la observación, la realización de entrevistas semiestructuradas y el análisis de datos mediante el software cualitativo Atlas.ti. Además, el uso conjunto de etnografía y técnicas cualitativas propiciaba ciertas ventajas de interés: un "mayor control de un conjunto de variables contextuales, posibilidad de elección de los informantes más adecuados, mayor facilidad de empatía con ellos, etc." (Romaní, 1999: 151).

Previamente al trabajo de campo se realizó una primera aproximación por medio de la búsqueda y análisis bibliográficos durante todo el año 2010, indagando en fuentes diversas para conocer el estado de la cuestión. Me centré, sobre todo, en trabajos sobre la atención, tratamiento y conceptualización de la patología dual, así como sobre las leyes, políticas sanitarias y decretos de ámbito estatal para poder enmarcar el contexto en su totalidad. Se vio que la literatura antropológica específica sobre el tema era escasa, aunque las referencias halladas pertenecientes a distintas materias fueron de gran utilidad para construir finalmente el cuerpo de análisis teórico.

En octubre de 2010 me introduje en el campo realizando la primera observación; en concreto, acudí a una reunión de coordinación entre los profesionales de salud mental, drogas, patología dual e instituciones penitenciarias. Tras esta inmersión inicial permanecí seis meses realizando observaciones y entrevistas en un Centro de Atención y Seguimiento. Durante este periodo asistí a reuniones de coordinación con diferentes dispositivos, reuniones de equipo y reuniones de formación. Aproveché, además, todo aquel periodo para entablar una gran variedad de conversaciones informales con todos los miembros del equipo. Se hicieron entrevistas en profundidad a siete de los profesionales de dicho dispositivo: una psiquiatra, un psicólogo, un antropólogo, una trabajadora social, una mediadora intercultural, una educadora social y un enfermero.

Posteriormente, tras un largo periodo de espera, logré concertar entrevistas con dos profesionales de una Unidad de Patología Dual, concretamente con uno de los psiquiatras de la unidad y con la trabajadora social.

A lo largo de la estancia en el campo me fui nutriendo de numerosas referencias bibliográficas, pertenecientes tanto al ámbito médico como al antro- 
pológico. Finalmente, el análisis de los datos se realizó mediante el software cualitativo Atlas,ti, de gran ayuda a la hora de realizar las transcripciones, codificaciones y la construcción final del presente artículo.

\section{Resultados}

\subsection{Salud mental y drogodependencias $¥$ dos redes encaminadas a la unificación}

\subsubsection{Marco jurídico-administrativo}

Los inicios del cambio en el modelo contemporáneo de atención en salud mental en Cataluña ${ }^{1}$ surgen de 1914 a 1923, cuando la Mancomunidad pretendió introducir en sus propuestas una cierta reforma de la salud mental, entre cuyos objetivos se encontraba la mejora de las condiciones de vida de los enfermos. Tras varios cambios, la llegada de la Guerra Civil cortó los aires de reforma, que volvieron a aflorar a finales de la década de 1960. A mediados de la década siguiente se impulsaron proyectos reformistas y se abrieron los primeros centros de Higiene Mental y dispensarios comunitarios desde algunos hospitales psiquiátricos, persiguiendo la idea de la sectorización ${ }^{2}$. A inicios de los años 80 se crean los primeros Centros de Atención Primaria en Salud Mental, concebidos como el primer peldaño en la atención a la salud mental. En 1981, la Comunidad Autónoma de Cataluña asume las transferencias de la Seguridad Social y se crea el mapa sanitario, abriéndose nuevos centros como los de atención a las drogodependencias. En 1985 y 1986 se crearon dos documentos importantes para la reforma en salud mental, la Reforma de la Asistencia Psiquiátrica y la Ley General de Sanidad, respectivamente, que trataban de conseguir una forma de atención en salud mental basada en redes de recursos sanitarios y comunitarios alejada del modelo anterior, apostando por un nuevo paradigma de atención inserto en la comunidad que procurara sostener y tratar al enfermo en redes de recursos sanitarios y sociocomunitarios interconectados. En los años

1. Alfred Capellà (2001) hace un recorrido por los inicios del cambio de modelo de atención en salud mental en Cataluña, en el que me baso para realizar este análisis introductorio.

2. Para conocer con mayor profundidad y riqueza de detalles el proceso del modelo catalán de la salud mental ver: Comelles, J. M. (2006), Stultifera navis. La locura, el poder y la ciudad. Lleida: Editorial MILENIO; y Comelles, J. M., (1988), La razón y la sinrazón. Asistencia psiquiátrica y desarrollo del Estado en España. Barcelona. 
90 las competencias de las diputaciones se transfieren a la Generalitat, la cual crea, a su vez, dos elementos básicos: el Servei Català de Salut y el Pla de Salut Mental, defendiendo el "modelo mixto" consistente en que el programa de salud mental ya no provee los recursos como acostumbraba el sistema público de salud, sino que tiene como finalidad la planificación, financiación, contratación y valoración de los recursos de salud mental, que pueden provenir tanto de lo público como de lo privado.

La culminación de este proceso fue el Decreto-ley que define la red de salud mental de utilización pública en julio de $1999^{3}$, cuya finalidad era integrar en una red única todos los servicios que realizan la atención en salud mental, advirtiéndose en el mismo decreto que su implantación sería progresiva debido a su complejidad.

En 2001 existían todavía redes paralelas — es decir, no se daba un funcionamiento en red-, y las unidades de atención a las toxicomanías (CAS) no estaban aún integradas en la red general. No fue hasta el año 2002 cuando se extendió la transferencia de las competencias en materia de sanidad al territorio INSALUD, dándose así los primeros pasos del cambio hacia el nuevo modelo de atención.

Dentro de este marco en el que se lucha para que la salud mental logre la posición dentro del sistema que se merece, y se obtenga un servicio de calidad para los usuarios, no se incluye todo lo referente al tratamiento de las drogadicciones. Como ya se ha comentado existían redes paralelas, y la salud mental y las drogadicciones se encontraban en redes separadas. El proceso que se ha seguido en Cataluña en materia de adicciones corre parejo al de la salud mental, pero presenta peculiaridades propias y leyes específicas ${ }^{4}$ que remarcan la diferenciación entre ambas "disciplinas".

3. La Ley de Ordenación Sanitaria de Cataluña 15/1990, de 9 de julio (LOSC), marco normativo y jurídico que establece en su disposición adicional sexta la integración de la asistencia psiquiátrica en el sistema de cobertura del Servicio Catalán de la Salud. Extraído del Plan Director de Salud Mental de Cataluña, págs. $15-16$.

4. Las normativas específicas promulgadas en el ámbito de las drogodependencias han sido:

1. Ley $1 / 2002$, de 11 de marzo, de tercera modificación de la Ley 20/1985, de 25 de julio, de prevención y asistencia en materia de sustancias que pueden generar dependencia.

2. Orden de 31 de octubre de 1985 del Ministerio de Sanidad y Consumo que regula los tratamientos con metadona dirigidos a toxicómanos dependientes de opiáceos y establece que se realizarán únicamente en centros o servicios sanitarios públicos o privados sin ánimo de lucro, autorizados por la Administración sanitaria de las CCAA. 
La Red de Atención a las Drogodependencias de Cataluña (XAD) se constituyó como una red específica y profesionalizada para dar atención integral a personas con trastornos relacionados con el consumo de sustancias psicoactivas, siendo esta red una de las prioridades de los planes de drogodependencias que se han ido elaborando en España desde 1986. El Parlamento de Cataluña aprobó la Ley 20/1985, de Prevención y Asistencia en materia de sustancias que pueden generar dependencia. Esta Ley, una de las pioneras en España, constituye el eje normativo vertebrador del conjunto de medidas del Plan Catalán de Drogodependencias; entiende la dependencia de las drogas como una enfermedad común que debe tratarse en el sistema sanitario. A raíz del surgimiento de esta Ley se vio la necesidad de desarrollar una acción coordinada y de asegurar la colaboración entre los diferentes departamentos del Gobierno de la Generalitat y las administraciones locales. Así, se constituyó la Comisión de Coordinación y de Lucha contra las Dependencias de Drogas y, al mismo tiempo, se crearon el Consejo Director y el Órgano Técnico de Drogodependencias (OTD), adscritos a la misma. De forma paralela se creó para toda España el PNSD, que en parte sirvió como coordinador, y en parte como órgano de financiación ${ }^{5}$.

A lo largo de dicho proceso se entendió la necesidad de promover la integración y unificación de las redes de salud mental y drogodependencias para mejorar su funcionamiento y la atención a los usuarios, motivo por el cual en

\footnotetext{
3. Decreto de $9 / 1986$ de 16 de enero, sobre procedimiento sancionador de la Ley 20/1985, de 25 de julio, de prevención y asistencia en materia de sustancias que pueden generar dependencia.

4. Decreto 69/1987, de 20 de febrero, por el que se detallan colas y otros productos industriales inhalables con efectos euforizantes o depresivos.

5. Decreto $247 / 1987$, de 20 de julio, por el que se regula la señalización de las prohibiciones de venta y suministro de tabaco y bebidas alcohólicas a los menores de 16 años.

6. Decreto 184/1990, de 20 de junio, de creación del Programa de Atención Especializada para el Tratamiento de Conductas Adictivas, dirigido a los internos de instituciones penitenciarias.

7. Real Decreto 75/1990, de 19 de enero, que regula los tratamientos con opiáceos a drogodependientes.

8. Orden de 20 de febrero de 1991, reguladora de los tratamientos que contempla el Real Decreto 75/1990, de 19 de enero.

9. Real Decreto 5/1996, de 15 de enero, que modifica el Real Decreto 75/1990.

Extraído del Plan Director de Salud Mental de Cataluña. Pág. 19
}

5. Plan Director de Salud Mental y Adicciones. Pág. 18 
2006 se crea el Plan Director de Salud Mental y Adicciones ${ }^{6}$, que se marcaba una serie de objetivos dirigidos a integrar ambos campos en uno solo ${ }^{7}$.

Tal y como apunta el Plan de Salud de Cataluña 2010, en la actualidad"las necesidades asistenciales son cubiertas por unos servicios de calidad que generan confianza en la ciudadanía, pero en los cuales hay aspectos que son susceptibles de mejora ${ }^{8 "}$. La integración de las redes de salud mental y adicciones posibilitaría satisfacer dicha necesidad, mejorando la atención a los usuarios y favoreciendo la mejora de la atención sanitaria.

Entre las propuestas estratégicas apuntadas por el Plan de Salud de Cataluña 2010 considero pertinente destacar la siguiente propuesta: "Salud mental y adicciones con un modelo más comunitario, con más capacidad de resolución y mayor soporte a la atención primaria, reconversión psiquiátrica e integración funcional de los servicios de salud mental y de drogodependencias", la cual hace patente una vez más la importancia de la unificación de ambas redes de atención sanitaria, considerándola como una de las tendencias principales en el desarrollo y la adecuación de los servicios de salud que señala el Mapa sanitario, sociosanitario y de salud pública en el horizonte del año 2015. El Departamento de Salud, mediante el Plan Director de Salud Mental y Adicciones, propone un nuevo modelo de atención para las personas de todas las edades que integra la promoción de la salud y la prevención, las actividades asistenciales y de rehabilitación, e impulsa la coordinación y cooperación entre los servicios de atención a las personas con un alto componente intersectorial.

Entre los objetivos del Plan de Salud de Cataluña 2002-2005 encontramos la Patología Dual, por estar considerada como uno de los problemas de salud 6. Decreto 30/2006, de 28 de febrero, por el que se crea el Plan Director de Salud Mental y Adicciones y su Consejo Asesor. Diario Oficial de la Generalidad de Cataluña, núm. 4584 - 02/03/2006. Págs. 9650-9652.

7. Plan Director de Salud Mental de Cataluña. Págs. 8, 9 y 10.

8. Plan de Salud de Cataluña 2010: “5 ejes estratégicos que articulan las políticas de salud”. Pág. 4. Este documento es la segunda parte del Plan de Salud, y en él se presentan las propuestas estratégicas del Gobierno de la Generalitat en materia de salud con vistas al año 2010, que se encuentran en la línea de las orientaciones y directrices de organismos y expertos nacionales e internacionales. La primera parte del Plan de Salud recibe el nombre de «Informe de salud en Cataluña. Evaluación de los objetivos de salud», y contiene el análisis de situación y la evaluación a mitad de periodo de los objetivos de salud fijados para el año 2010. Dicho Plan de Salud, de acuerdo con lo que establece el Plan de Gobierno 2007-2010, es la agenda estratégica del Gobierno de la Generalitat de Catalunya por lo que respecta a la salud, y pretende integrar y dar coherencia a las políticas de salud y servicios de los ámbitos sanitarios y sociales implicados en la salud, así como añadir una visión amplia allí donde los aspectos socioeconómicos y los determinantes de la salud cobran tanta importancia como las actuaciones preventivas y asistenciales.

9. Plan de Salud de Cataluña 2010. "5 ejes estratégicos que articulan las políticas de salud”. Pág. 59.

Arxiu d'Etnografia de Catalunya, n. ${ }^{\circ}$ 12, 2012 
de gran magnitud e impacto ${ }^{10}$. Figuraba entre las patologías más importantes incluidas en los trastornos mentales la coexistencia de dependencia de sustancias y otros diagnósticos psiquiátricos — conocido como diagnóstico dual, que está considerado como un importante problema en nuestro medio ${ }^{11}$.

Tras conocer el marco jurídico-administrativo y situarnos en el contexto en el que se enmarca la salud mental junto con las adicciones en el ámbito sanitario catalán, pasamos a ver cuál es la realidad que se palpa en los centros sanitarios especializados en la atención a drogas y salud mental; y qué mejor forma de conocer esta realidad que a través de los propios profesionales de salud que en ellos trabajan.

3.1.2 De la teoría a la práctica; la unificación de las redes en el trabajo diario de los profesionales

De todo este proceso que hemos relatado con anterioridad, los profesionales entrevistados reflexionan al respecto.

La narrativa de $\mathrm{Pau}^{12}$ refleja que el proceso para los profesionales implicados está siendo largo: "Hace 10 o 12 años, y aún estamos así, acercándonos [...], es un proceso que parece que es imparable, lo que pasa es que va muy poco a poco. No sé cómo evolucionará”. Él lleva muchos años trabajando en este sector y ha vivido desde su inicio esta evolución hacia el cambio de paradigma. Participó en sus inicios y en la reunión de expertos que daría pie al Plan Director, viviendo así desde dentro y como parte activa el proceso de modificación que aún sigue desarrollándose: "Fue hace 10 o 12 años que hubo un encuentro de CAS de Cataluña [...] fue como el embrión de lo que después llamarían el Plan Director de Salud Mental y Adicciones, y allí fue donde primero yo vi planteado que era un poco absurdo que hubiera una red de drogodependencias y una red de salud mental, porque todos estábamos haciendo salud mental [... ]. Entonces se empezó a hablar que se tendrían que fusionar las dos redes". En su discurso queda de manifiesto que está de acuerdo con que se lleve a cabo dicha unificación, aunque por eso mismo también critica el excesivo tiempo empleado en lograr el cambio real: "La idea de que haya una sola red de salud

10. Plan Director de Salud Mental y Adicciones. Pág. 27.

11. Plan Director de Salud Mental y Adicciones. Pág. 37.

12. Psicólogo del CAS. 
mental y drogas me parece más coherente que no el hecho de que haya dos redes paralelas haciendo más o menos lo mismo [...]. Claro, yo, por una parte, pienso que por lógica, por coherencia, tendría que haber una sola red porque es todo lo mismo, pero en la práctica.... Pau comenta la necesidad de que se lleve a cabo la unificación para lograr mejor coordinación y mejores resultados, aunque expone también sus miedos al respecto, referentes a la pérdida de recursos y a un posible empeoramiento de los servicios: "El miedo que tengo es que los que tengan más recursos que no los pierdan [...], lo que me sabría mal es que el hecho de fusionarse las dos redes significara para nosotros perder recursos o capacidades". Encontramos en su discurso la actual dificultad de coordinación entre los profesionales de salud mental y los expertos en drogadicciones; los primeros, según comenta el propio Pau, no llegan a comprender, a pesar de las reuniones de coordinación, cuál es la labor exacta que desempeñan sus compañeros en materia de drogas. "Vale, nosotros trataremos depresión y vosotros alcoholismo y ya está", le comentan los de CSM. Este discurso está también presente en las narrativas de varios compañeros suyos del CAS, como es el caso de Clara ${ }^{13}$ : Encontramos muchos conflictos trabajando con Salud Mental [... ]. Te dicen: 'mira a este paciente, yo le estoy tratando el trastorno depresivo, pero como de vez en cuando bebe, tú trátale el alcohol'. No sé, no nos entienden, entendemos más a los pacientes que no a los compañeros de Salud Mental [... ]. No nos aclaramos entre los profesionales".

Clara opina también que ambas redes deberían estar integradas en una sola: "La red yo creo que ha de ser integradora, porque un usuario cuando llegue aquí no puede ir a dos servicios, tiene que estar integrado todo en un mismo servicio", y que desde ambos lugares se debería tratar la patología dual por el bien de los profesionales y de los pacientes, ya que este paralelismo y separación perjudica a todos (usuarios y profesionales de drogodependencias) y no beneficia a nadie.

Como vemos, en la narrativa de Clara el encuentro entre profesionales de la salud en cuanto al modo de entender la atención de las personas que presentan patología dual está lejos de darse con facilidad. Los profesionales de los dispositivos de SM tienden a derivar los pacientes que presentan tóxicos hacia los CAS, para que allí les traten exclusivamente la drogodependencia, con lo

13. Clara, psiquiatra del CAS. 
que se hace patente la incomprensión que existe en el modo de trabajar de cada dispositivo. Clara achaca este rechazo al tipo de paciente y sus características, puesto que, según comenta, son más difíciles de tratar que el tipo de paciente estándar al que están acostumbrados en salud mental:

En Centros de Salud Mental que están acostumbrados a trabajar con pacientes no quieren tóxicos, vuelven a ser aquellos que rechazan porque se confrontan, porque ellos están muy acostumbrados a las personas psicóticas [...] pero las personas con trastorno de personalidad y las personas drogodependientes son personas que si tú no negocias con ellas te levantarán la voz, o dirán que no les parece bien y no callan, y eso es lo que se hace difícil de llevar ${ }^{14}$.

Estas opiniones las comparte Zimberg (1996: 21), al afirmar que "con frecuencia se ha rechazado su admisión a tratamiento y se han desviado hacia otros programas o simplemente se los ha ignorado por considerarlos intratables o de mal pronóstico".

La unión entre las dos redes no solo se encuentra en proceso en los ámbitos administrativo y jurídico, sino también, como vemos, en el modo de entender el trabajo entre los profesionales que trabajan en ambas redes, puesto que si no existe una verdadera comprensión del trabajo para coordinarse de modo eficiente e integrador, los resultados no serán los esperados. A pesar de los puntos de desencuentro, vemos en el discurso de Clara que algo ha empezado a cambiar gracias a este proceso modificador, en gran parte por la creación de la Unidad de Patología Dual. Clara comenta: "Hay cambio y no lo hay. Se ha creado una unidad de patología dual [...]. Cuando antes no estaba la unidad de patología dual, hace 4 años que existe aquí, nosotros queríamos ingresar un paciente y nada más veían que tomaba tóxicos no lo ingresaban".

Carlos, antropólogo de un equipo multidisciplinar de drogodependencias, reflexiona sobre la UPD como uno de los intentos de unificar las redes de salud mental y adicciones dentro de las innovaciones posibilitadas por el Plan de Salud Mental y Adicciones: "La red de drogas y la red de salud mental siempre han estado separadas [...] es uno de los primeros intentos de juntar las redes y depende del lugar donde eso pase también se entienden más o menos, y como nunca de la vida se han entendido [...] esta unidad está abriendo grandes oportunidades de trabajo conjunto entre las redes".

14. Idem. 
Consideran, así pues, que los profesionales de la UPD están más próximos a la comprensión de la realidad del trabajo de ambas redes, lo que constituye un impasse beneficioso para lograr los objetivos planificados de integración de la salud mental y las adicciones; un integración cuyos principales beneficiados serán todos aquellos pacientes que presentan patología dual, porque por fin podrán gozar de una atención integral de calidad. Esta unidad es, así pues, un paso adelante hacia el cambio y la unificación de las redes que puede ayudar a llegar a un mejor entendimiento entre los profesionales de ambas, que como él mismo remarca - y otros compañeros suyos - nunca se han acabado de entender. Esta nueva unidad se valora de forma positiva por promover oportunidades de trabajo conjunto.

Respecto a la coordinación anunciada en el Plan, Carmen - educadora social del CAS - comenta:"Realmente no está, a ver, se hacen coordinaciones, y sí, se intentan hacer, pero si se hace una vez cada tres meses en un caso puntual [...] es complicado. Hay cosas que se han mejorado bastante, pero hay cosas que siguen fallando muchísimo, y son estos pacientes que van de aquí hacia allá, y se les dice 'no, ahora hemos decidido entre los dos equipos que es mejor que te trates aquí, tendría que ser mucho más fluida esta relación".

Carmen también presenta en su discurso los dos polos antes vistos en sus compañeros. Por un lado reconocen que algunas cosas han cambiado, pero por otro siguen quejándose de la situación y consideran que las cosas deberían ser mejores, haciendo referencia una vez más a los "pacientes a la deriva" que quedan a merced de las decisiones de los profesionales, que para más inri no acaban de entenderse entre ellos.

Vemos así que, aunque diversos aspectos de la práctica psiquiátrica y de la adicción son semejantes, existen también barreras entre ambos campos. Y son los pacientes con patología dual los que se ven afectados en mayor grado por esta separación, al ser desviados de un sistema a otro (Zimberg, 1996). La unificación de redes se dibuja, entonces, como el mejor fin para la atención adecuada a esta población, que además cada día va más en aumento. 


\subsection{La nosología incierta: Nuevas etiquetas para los nuevos tiempos}

En los últimos años ha aumentado la preocupación sobre el diagnóstico y el tratamiento de los pacientes con patología dual. Existe una cierta ignorancia sobre estos pacientes, pese a su inclusión en programas de atención psiquiátrica y drogodependencias; se ha observado un aparente aumento de la prevalencia de los pacientes con trastornos duales (Zimberg, 1996), lo cual ha contribuido a que el diagnóstico dual se erija cada vez más como un área prioritaria. "Esta comorbilidad, incuestionable en ciertos casos, viene constatándose en múltiples y recientes estudios, por lo que supone un reto llegar a conseguir un consenso en torno a aspectos fundamentales relacionados con lo que se ha denominado Patología Dual" (Asociación DUAL). El mismo término de comorbilidad ya supone de por sí un indicador de la dificultad para lograr un consenso, ya que la comorbilidad no es otra cosa que "la existencia de diferentes criterios diagnósticos en un mismo caso y la dificultad de discernir qué pertenece a qué" (Martinez-Hernaez, 2000: 268).

A pesar de haberse colocado en los últimos años como uno de los problemas de salud de gran magnitud e impacto ${ }^{15}$ — está considerado un problema de importancia en nuestro medio-, resulta de interés poner de relieve el hecho de que el término Patología Dual no es más que una "nueva” etiqueta diagnóstica pensada para clasificar a un "nuevo" tipo de pacientes siguiendo la orientación biomédica en psiquiatría (o neokraepelinismo), y especialmente la tendencia característica de ésta de poner el énfasis en la clasificación (Martinez-Hernaez, 2000).

Es un cajón de sastre en el que se engloban multitud de combinaciones posibles entre trastornos por abuso de sustancias y trastornos mentales, y que ha generado un gran debate y controversia. Profesionales y entidades no han llegado a un acuerdo unánime sobre una definición objetiva de esta patología.

Tal y como defienden Edward V. Nunes Jr. y Deborah A. Deliyannides, "el extenso campo de coexistencia de los trastornos por uso de sustancias con otros trastornos mentales contiene un gran número de interrogantes no resueltos y de aspectos susceptibles de investigación. Sin embargo, los intentos realizados para desarrollar un abordaje de investigación sistemático en este

15. Plan Director de Salud Mental y Adicciones. Pág. 27. 
tema se enfrentan con dos aspectos complejos: las numerosas interrelaciones existentes entre el trastorno por abuso de sustancias y el trastorno mental, y el número absoluto de posibles combinaciones entre ambos trastornos. [...] La simple enumeración de los trastornos mentales y de sustancias más frecuentes ya indica, en cierta manera, las posibles y numerosas combinaciones existentes entre ambos trastornos" (Nunes y Deliyannides, 1996: 321).

La Patología Dual se entiende, así pues, como un fenómeno complejo y controvertido ${ }^{16}$. Décadas de documentada investigación sobre esta comorbilidad no han impedido que aún siga siendo no solo un tema polémico, sino también negado, cuando no desconocido, por algunas de las corrientes clínicas que se ocupan de estas patologías. Existen numerosos relatos de casos de sujetos no diagnosticados, o sin un tratamiento adecuado, lo que incide en la morbilidad y mortalidad y, en definitiva, en un mayor sufrimiento para los pacientes y sus familias ${ }^{17}$. En la actualidad hay evidencias que señalan que un número importante de personas sufre simultáneamente trastornos adictivos y psiquiátricos ${ }^{18}$.

Desde la $\mathrm{SEPD}^{19}$ defienden que esta nueva realidad clínica necesita no solo conocer los mecanismos que subyacen a esta comorbilidad, sino también disponer de un espacio común para los profesionales implicados que dé soporte a las inquietudes y proyectos investigadores, clínicos, docentes, etc. Pero, ¿cómo se entiende este término desde las diferentes asociaciones y profesionales sanitarios especializados en el tema?

16. Asociación DUAL. Esta Asociación se define a sí misma como una entidad pionera en la creación de programas, servicios y centros específicos para el colectivo de personas afectadas por una Patología Dual, como también para sus familiares y los profesionales vinculados a su tratamiento. La Asociación se creó en junio del año 2000, y comenzó a gestionar en agosto del mismo año el primer piso-residencia para la rehabilitación integral de personas afectadas por la Patología Dual. Actualmente, esta Asociación, a través de un contrato de Gestión de Servicio Público con la Agencia Antidroga de la Comunidad de Madrid, gestiona un piso de apoyo al tratamiento en Madrid, así como un piso supervisado de continuidad en régimen de autogestión con apoyo clínico y educativo.

17. Carta de presentación del presidente de la SEPD (Sociedad Española de Patología Dual), Nestor Szerman.

\section{Asociación DUAL.}

19. La Asociación Española de Patología Dual (SEPD), tal y como comenta su presidente Nestor Szerman, surge en 2005 y se constituye en un espacio común para los clínicos, médicos y psicólogos que trabajan en distintos dispositivos de atención a drogodependientes, de psiquiatría y salud mental, y otros lugares donde encontramos a pacientes con patología dual. Pretenden y defienden una asociación - que agrupa a más de 900 profesionales, fundamentalmente españoles pero también hispanoamericanos, de distinta formación - que supere antiguas barreras, jerarquías y suspicacias, y que al mismo tiempo sea independiente de las administraciones sanitarias gubernamentales, de los intereses comerciales de la industria y también de las legítimas reivindicaciones profesionales, ya organizadas en otras instituciones. 
En la presentación del Congreso Internacional de Patología Dual: Conductas Adictivas y Otros Trastornos Mentales, celebrado en mayo de 2008, definían la Patología Dual como "la concurrencia de trastornos por Uso de Sustancias y Otros Trastornos Mentales" ${ }^{20}$, considerándola como una "nueva entidad clínica".

La SEPD la define en sus estatutos como la "concurrencia en una misma persona de una conducta adictiva (alcoholismo, toxicomanías, adicciones comportamentales, etc.) y un trastorno mental". En cambio, la Asociación DUAL hace una definición que, a pesar de presentar ciertos matices comunes, muestra también algunas variaciones: "asociación cruzada (coexistencia o comorbilidad) de dos situaciones clínicas: la presencia de un trastorno mental por un lado $y$, por otro, el uso patológico de sustancias tóxicas (consumo o abusos inadecuados, así como dependencia de drogas)".

Ambas asociaciones definen la presencia de un trastorno mental en dicha patología, pero la parte correspondiente al abuso de sustancias varía a la hora de expresarse. La primera asociación tiene en cuenta adicciones comportamentales - lo cual nos abre un gran abanico de posibilidades indefinidas e infinitas-, y además deja un etcétera al final de dicho paréntesis que deja entrever la no especificidad y falta de acotación de dicha patología. La segunda asociación, en cambio, no comenta nada a este respecto, apuntando en su paréntesis una frase de "objetividad" ambigua: "consumo o abusos inadecuados". ¿En qué momento el consumo de una droga se considera adecuado y por tanto no forma parte de esta patología? Se observa, así pues, cómo a pesar de tratarse de dos asociaciones especializadas en la atención a este trastorno, el acuerdo en una definición del término queda lejano, y las definiciones, lejos de acotar y delimitar qué es y qué no es Patología Dual, lo que hacen es un mero intento de definición que no logra un resultado unánime que permita el consenso objetivo.

La falta de consenso en la definición de la nosología resulta un tanto inquietante. ¿Cómo puede existir una categoría diagnóstica psiquiátrica que sea definida de dos modos diferentes? La clave reside en el hecho de que dicha nosología no se encuentra estipulada en ninguna edición del DSM, y tampoco se encuentra reconocida en la CIE-10. Así pues, si no está preestablecido obje-

20. Está definida del mismo modo en la Invitación al Congreso Internacional de Patología Dual que se celebrará este mismo año de 2011 en la ciudad de Barcelona. 
tivamente en los manuales de referencia es normal que se dé falta de consenso al respecto entre los distintos profesionales y entidades.

Nos encontramos ante una situación peculiar o extraña, en la que topamos con una nosología que no se encuentra regulada en los manuales de referencia de la psiquiatría, pero que sin embargo los profesionales emplean para aludir a una comorbilidad específica. Esta etiqueta constituye un claro ejemplo de la idea que defiende Ángel Martínez en Anatomía de una ilusión cuando afirma que "una categoría diagnóstica no es percibida como una construcción social más o menos arbitraria, que se caracteriza por su inscripción en un contexto cultural e histórico determinado, que es el resultado de formas históricas de tratamiento" (Martínez-Hernáez, 2000: 267), y el mismo autor comenta además cómo "desde la psiquiatría biomédica, las categorías son entendidas como unidades reales y universalmente válidas" (Martínez-Hernáez, 2000: 267). No puede negarse que esta categoría diagnóstica ha sido fruto de una construcción social arbitraria en un contexto cultural e histórico determinado; así pues, estamos ante una nosología que, lejos de la "objetividad", refleja la fragilidad de las etiquetas diagnósticas de un modo claro. ¿Cómo podrían defender la validez universal de esta etiqueta diagnóstica si ni siquiera se encuentra definida oficialmente en los manuales?

Ante esta falta de unanimidad en la definición, ¿cómo trabajan los profesionales especializados en su día a día? ¿Qué discursos o qué comprensión tendrán de lo que significa dicha nosología? A la hora de abordar este tema resultaba esencial plantear una pregunta que permitiera conocer cuál era la definición que los profesionales entrevistados tenían sobre Patología Dual, para poder así analizar el discurso de cada uno de ellos. Esta pregunta clave fue: ¿qué es la Patología Dual?, y entre las respuestas encontramos una amplia variedad, que pasaré a comentar y analizar a continuación.

Neus, trabajadora social, la define del siguiente modo: "Hasta hace unos años no se ha definido eso de la Patología Dual [... ] pero ahora [... ] ya se hace esta distinción, pero las personas que consumen sustancias ya se sabe que pueden acabar con problemas de salud mental ${ }^{21 "}$.

Neus lleva 20 años trabajando en este ámbito y reflexiona sobre la "novedad" del término; novedad aparente: no es que haya surgido una nueva enfermedad, sino que simplemente nunca se había nombrado como tal, es decir, que 21. Neus, trabajadora social del CAS. 
lo novedoso es la etiqueta y no las características de las personas "portadoras" de la misma.

\section{Clara, psiquiatra del CAS, comenta:}

Un problema de adicción y un trastorno psiquiátrico, la suma de los dos. El término de Patología Dual surgió haciendo referencia a la suma de un trastorno adictivo más un trastorno psiquiátrico, lo que pasa es que así para ir por casa, después se inventaron que una persona con un trastorno mental y una psicosis pues también es un trastorno dual, que también lo es, pero que si vamos inventando cosas así también podríamos decir que tener una fractura en un momento dado y tener un trastorno depresivo también es un trastorno dual [...]. Diría que no les gusta el término de trastorno dual precisamente porque puede crear discrepancias ${ }^{22}$.

La ambigüedad está presente en el término, ya que puede hacer referencia a cosas distintas, lo cual crea discrepancias entre profesionales, aunque existe cierto "consenso" en entender por dual la combinación de un trastorno adictivo y uno mental. Siguiendo en cierta manera el discurso de Neus, Clara alude también al surgimiento de esta nosología, haciendo hincapié en que se trata de un término acuñado recientemente. Resulta clave en el discurso de Clara la frase en la que remarca que es un término "para ir por casa”, es decir, que no se encuentra validado en los manuales de psiquiatría.

Marcos, psiquiatra de la UPD, la define escuetamente: "Los criterios son: abuso, dependencia o uso de sustancias, y un trastorno metal"23, aunque se extiende más en su definición de paciente dual: "el hecho diferencial es que han de tener un trastorno por uso de sustancias. Patología Dual también podrían ser los pacientes con patología mental y dependencia al tabaco, entonces están todos, porque pacientes psiquiátricos, la gran mayoría, el 70,75\%, son fumadores, entonces [...] lo que clásicamente se entiende son tóxicos con consumo de sustancias psicotrópicas ilegales, o alegales" 24 .

Carmen, educadora social del CAS, da una definición semejante a la de sus compañeros: "una persona que tiene problema de tóxicos y tiene problema de enfermedad mental, y que los tóxicos influyen en la enfermedad mental y la

22. Clara, psiquiatra del CAS.

23. Marcos, psiquiatra de la UPD.

24. Marcos, psiquiatra de la UPD. 
enfermedad mental influye sobre el consumo de los tóxicos, ¿no? y que todo va un poco ligado" 25 .

Se observa un cierto consenso entre lo que los profesionales entienden por dual, en el sentido de que en sus discursos la presencia de un trastorno de tóxicos en comorbilidad con un trastorno mental es la guía para encajar en esta etiqueta. En el discurso de Marcos se observa el cajón de sastre que es esta categoría diagnóstica, ya que si somos fieles a las definiciones, tal y como él comenta, el $75 \%$ de pacientes psiquiátricos que son fumadores podría estar englobado dentro del marco de pacientes duales. Así pues, tenderían más bien a entender por duales a aquellos que abusan de sustancias alegales o ilegales - no al tabaquismo- dentro de los trastornos adictivos que forman uno de los polos de dicha dualidad patológica.

Esta omisión del tabaquismo dentro de la consideración de los trastornos adictivos conformadores de uno de los polos de la patología dual resulta curiosa, a la vez que interesante. ¿Cuáles son los motivos que libran a los fumadores de encajar dentro de esta etiqueta diagnóstica? ¿Por qué liberar al 75\% de los pacientes psiquiátricos de "la dualidad"? Si el tabaco fuera tomado en cuenta, muy poca gente, solo un $25 \%$ de enfermos mentales, quedaría exenta de un diagnóstico dual, lo que quizás crearía un alarmismo exacerbado que podría llevar a cuestionar la utilidad de las etiquetas diagnósticas, puesto que lo "normal" sería ser "dual".

Pep, enfermero del CAS, expone su opinión sobre el término: "es una persona con dos problemas, uno psicopatológico y un problema de consumo de sustancias ${ }^{26 "}$. De entrada está de acuerdo con el "consenso" que hemos observado en el resto de entrevistados, pero hace posteriormente un matiz que va más allá: "soy de la parte de aquí del servicio que cree que todos los pacientes tienen algo de dual, por un lado o por otro siempre sale alguna cosita, hay casos muy marcados que se evidencia rápido, que hay algún problema, alguna patología psiquiátrica o enfermedad mental [...] y enfoca su problema en las sustancias $^{27}$ ". Más adelante hizo hincapié en el auge que está teniendo este etiquetaje, debido, según él, a una mayor evidencia: "eso de Patología Dual tampoco es tan nuevo ni tan antiguo [... si te paras a pensar... ¡uf!, la mayoría de pacientes

25. Carmen, educadora social del CAS.

26. Pep, enfermero del CAS.

27. Idem. 
podría llevar la etiqueta colgada desde el primer día. Creo que se habla más de Patología Dual pero no porque lleguen más sino porque es más evidente para la gente, que la dualidad es real y está en mucha gente, se habla más pero no porque haya habido una infección de duales ${ }^{28}$ ". Considera que la dualidad está presente en todos los pacientes que les llegan; incluso en el momento final de la entrevista comenta la intranquilidad que experimenta en ocasiones al plantearse su propia dualidad en ciertos momentos: "Cuando salgo de fiesta por ahí y me emborracho, me lo planteo a veces, ¿no?, porque todos tenemos nuestras cosas [...] y en ese momento además con un consumo o a veces un abuso de alcohol... qué me diferencia a mí de ellos? Lo único que me salva es que yo no tengo puesta la etiqueta ${ }^{29 "}$.

Pau, psicólogo del CAS, la define de modo semejante a Pep:

Soy un poco crítico en el sentido de que pienso que todos somos duales, los problemas con drogas [...] los vemos más como un síntoma de algún otro trastorno o conflicto más profundo que se expresa de esta manera que no como un trastorno en sí [...]. La precisión ésta de que solo a algunos se les llama duales y a otros no, pues en realidad es porque el otro trastorno que hay detrás es un trastorno descrito y etiquetado [...] , en la práctica con lo que me encuentro es que siempre hay más cosas ${ }^{30}$.

La dualidad, por tanto, es común a todos ellos; el matiz reside en la etiqueta que se le pone a la persona. Pau va un paso más allá que Pep y reflexiona en torno a aquello que hay detrás. Considera que siempre hay algo detrás, teniendo en cuenta las variables de contexto social, familiar, etc., que desempeñan un papel importante en el surgimiento o desarrollo de las patologías.

Neus, trabajadora social del CAS, hace una crítica que va en cierta consonancia con la observación de Pau sobre las demás variables que entran en juego: “:por qué dual y por qué no tri, o cuatri, o poli? [... es lo que decimos nosotros, las circunstancias del individuo, la sustancia y el entorno ${ }^{31 "}$. Aporta una visión más integral que no culpabiliza al sujeto de su enfermedad, y tiene en cuenta el contexto que le rodea como un factor importante para el desarrollo de su día a día y como innegable influencia en su enfermedad. El término dual resulta,

28. Pep, enfermero del CAS.

29. Idem.

30. Pau, psicólogo del CAS.

31. Neus, trabajadora social del CAS. 
de este modo, insuficiente ante las influencias externas y otras variables a tener en cuenta.

Carmen, educadora social del CAS, tiene en cuenta la función que los consumos desempeñan en la vida del paciente, además del contexto social, familiar, etc.: "puede ser a veces que el consumo sea un mecanismo de defensa, mi vida y todo lo que tengo alrededor está tan deteriorado que [...] bebo dos litros de vino y las cosas están ahí pero no hacen tanto daño ${ }^{32}$ ". Es decir, que el consumo sería una vía de escape para huir de una realidad en la que no quieren estar, un mecanismo para evadirse del mundo, desaparecer por unos instantes y mitigar el dolor. Al final, Carmen defiende la misma idea que su compañera al exponer que la dualidad queda corta para definir una realidad con problemáticas tan dispares y variadas, y propone nomenclaturas más amplias: “'Y por qué es dual? Porque se dejan muchas cosas [...] trial, trial, o decathlon, que dice la psiquiatra del CAS. ${ }^{33}$.

Carlos, antropólogo del CAS, comenta:

La Patología Dual es eso, pues darle nombre a determinados pacientes, que no solo tienen problemas graves con drogas, sino que también salta a la vista que tienen problemas también más allá de lo normal de nivel mental, pero bueno, casos de estos puros de decir 'mira, es un esquizofrénico cocainómano', muy poquitos he visto. No identificaría pacientes que es un Patología Dual [...] pues yo creo que se identifica más por el tipo de recurso ${ }^{34}$.

Se observa en su narrativa la importancia que otorga al hecho de no etiquetar al paciente bajo esta "nueva" nosología, defendiendo que no ha de ser un nuevo modo de referirse a las personas, sino más bien a los nuevos recursos y unidades de atención especializadas, huyendo de la clasificación de los usuarios para intentar evitar que el estigma de esta población siga creciendo al adherírsele nuevas formas de denominarlos.

Laia, trabajadora social de la UPD, define así a los pacientes duales: "es un paciente muy estigmatizado, muy rechazado y poco comprendido [...], creo que el paciente no se tiene en cuenta [...], se etiqueta y en función de la etiqueta se decide [... no podemos pretender clasificarlos [...] a mí el tema de diagnós-

32. Carmen, educadora social del CAS.

33. Carmen, educadora social del CAS.

34. Carlos, antropólogo del CAS. 
tico [... nos cuesta [...] porque a veces el hecho de poner una etiqueta a una persona lo marcas de por vida ${ }^{35}$ ".

Encontramos en estos dos últimos discursos una preocupación especial por no caer en el etiquetaje y en la estigmatización de estas personas con doble patología. Ambos manifiestan la magnitud de las secuelas de por vida que acarrea una etiqueta cargada de prejuicios, porque resulta aún más perjudicial el que cataloguen a una persona con una doble etiqueta estigmatizadora que califica con la doble cronicidad vitalicia: locura y drogadicción. Se percibe en sus discursos cautela a la hora de realizar diagnósticos, un intento de huir de clasificaciones innecesarias por el bien del usuario.

\section{Conclusiones}

Tras analizar el proceso de reforma sanitaria en Cataluña se ha observado cómo la unificación de las redes de salud mental y adicciones planificada durante tanto tiempo, y teóricamente ya implementada, aún no se hace patente en la práctica sanitaria diaria, por lo que queda todavía mucho camino por recorrer para poder hablar de la fusión real entre ambas. La apertura de la Unidad de Patología Dual y las reuniones de coordinación entre los diferentes equipos son los primeros indicios del cambio, que los profesionales valoran como positivos, puesto que facilitan su trabajo al abrir oportunidades de trabajo conjunto entre redes, y mejoran la atención a los usuarios, evitando en gran medida la proliferación de los "pacientes a la deriva" itinerantes entre ambos dispositivos.

El logro de un cambio real que posibilite la unificación de ambas redes pasa por conseguir un buen entendimiento entre los profesionales de salud mental y drogas, que en la actualidad no se da como debiera. La falta de entendimiento entre profesionales aparece, así pues, como un problema en la práctica diaria, un problema que sería interesante investigar en profundidad en futuros estudios mediante el análisis de las narrativas de todas las partes implicadas, para dar así una visión global e integradora que nos permita entenderlo en profundidad.

En cuanto a la definición de patología dual, se observa un cierto consenso entre profesionales y asociaciones especializadas a la hora de definirla como la concurrencia de un trastorno mental y uno adictivo, aunque existen numerosas variaciones que hacen que no se acabe de lograr una unanimidad sobre lo que

35. Laia, trabajadora social de la UPD. 
implica padecer esta patología. Varios de los profesionales han manifestado en sus narrativas dos temas de análisis que incitan al debate y a la reflexión: la opinión de que todos somos duales, y la necesidad de tener en cuenta más factores aparte de la salud mental y el consumo de drogas, como son los factores sociales, familiares, económicos, etc., los cuales, a pesar de que habitualmente no se toman en cuenta, forman parte de un todo unificado que no es, en ningún caso, independiente de los dos primeros. Así, en última instancia se proponen nosologías más amplias, como "triales" y "decathlon", lo que demuestra la importancia que tiene para estos profesionales observar qué hay más allá y no quedarse solo con lo evidente.

Se observa, por lo tanto, que estamos ante una nosología de "ir por casa", que se asemeja a un cajón de sastre en el que todo cabe según se mire, y que ha sido fruto de una construcción social que podemos calificar de arbitraria —al observarla desde la lógica conceptual, pero que tiene una explicación histórica sólida - en un contexto cultural e histórico determinado, lo que refleja la fragilidad de las etiquetas diagnósticas.

El auge actual de esta patología no se debe a un aumento de pacientes afectados, sino a un aumento de personas diagnosticadas, lo que ha causado una mayor visibilidad. La novedad reside en el término, en la etiqueta, y no en las características y síntomas de las personas "portadoras" de la misma.

Se deja entrever cierta preocupación por parte de los profesionales en referencia al etiquetaje y la consiguiente estigmatización hacia los pacientes que lleva ligada esta "nueva" etiqueta. Resulta, por tanto, de interés seguir esta línea de investigación sobre el estigma de este colectivo para analizar qué repercusiones acarrea esta categoría diagnóstica sobre los sujetos diagnosticados con ella, tanto en el ámbito social como en el trato que los profesionales especializados en su tratamiento les brindan — en la atención recibida y, por tanto, en su salud y bienestar-. ${ }^{36}$

\footnotetext{
36. Agradecimientos:

A mis tutores: Oriol Romaní y Àngel Martinez, por ayudarme, guiarme y apoyarme durante este trayecto. No podría haber estado en mejores manos.

A mis informantes: 1@s profesionales del CAS, por dedicarme su tiempo y compartir conmigo sus saberes y experiencias, siempre con una sonrisa.
} 


\section{Bibliografía}

Becker, H. S. (1964) The other side. Perspectives on deviance. New Cork London: The Free Press-Collier Macmillan Ltd.

Borràs i Cabacés, T. y Sardì i Garcia, A. (2005) "Cambios sociales, cambios en los tratamientos". Uso de drogas y drogodependencias. Monografia $\mathrm{Hu}$ manitas. (5): 119-137.

Capellá Batista-Alentorn, A. (2001) "La reforma en salud mental en Cataluña: el modelo catalán". Revista de la Asociación Española de neuropisquiatría 79 (Vol. XXI): 102-128.

Comelles, J.M. (2006) Stultifera navis. La locura, el poder y la ciudad. Lleida: Editorial MILENIO.

Comelles, J.M. (1988) La razón y la sinrazón. Asistencia psiquiátrica y desarrollo del Estado en España. Barcelona.

Decreto 30/2006, de 28 de febrero. Diario Oficial de la Generalidad de Cataluña Núm. 4584 - 02/03/2006: 9650-9652.

Erikson, K. T. (1964) "Notes on the sociology of deviance". En Becker, H. $\mathrm{S}$. The other side. Perspectives on deviance. New Cork - London: The Free Press-Collier Macmillan Ltd.

Goffman, E. (1964) Estigma. La identidad deteriorada. Buenos Aires - Madrid: Amorrortu.

Llei de Salut Pública. Butlletí Oficial del Parlament de Catalunya. 26 d'Octubre de 2009. Núm. 555

Martínez-Hernáez, A. (2000) “Anatomía de una ilusión. El DSM-IV y la biologización de la cultura" En: Medicina y cultura: estudios entre la antropología y la medicina. Bellaterra: 247-275.

Nunes Jr., E. V.y Deliyannides, D. A. (1996) “Investigación en diagnostico dual” En: Solomon, J., Zimberg, S., Shollar E. (eds) En: Diagnostico Dual: Evaluación, tratamiento, educación sanitaria y desarrollo de programas. Barcelona: Ediciones en neurociencias: 321-341.

Parsons, T. (1999) “Estructura social y proceso dinámico: el caso de la práctica médica moderna" En: El sistema social. Madrid. : 401-445.

Phillips, P. (1998) "The mad, the bad and the dangerous - harm reduction in dual diagnosis" International Journal of Drug Policy .9

Plan Director de Salud Mental de Cataluña. 
Plan Director de Salud Mental y Adicciones.

Plan de salud de Cataluña 2010، "5 ejes estratégicos que articulan las políticas de salud".

Romaní, O. (1999). Las drogas. Sueños y razones. Editorial Ariel. Barcelona.

Yang L. H., Kleinman A., Link B. G., Phelan J. C., Lee S., Good B. (2007) "Culture and stigma: Adding moral experience to stigma theory" En: Social Science E Medicine. 64 : 1524-1535.

Zimberg, S. (1996) "Introducción y conceptos generales del diagnostico dual" En: Solomon, J., Zimberg, S., Shollar E. (eds). Diagnostico Dual: Evaluación, tratamiento, educación sanitaria y desarrollo de programas. Barcelona: Ediciones en neurociencias : 21-40. 